\title{
Joint prediction of breast cancer histological grade and Ki-67 expression level based on DCE-MRI and DWI radiomics
}

\author{
Ming Fan, Wei Yuan, Wenrui Zhao, Maosheng Xu, Shiwei Wang, Xin Gao, and Lihua Li, Member, IEEE
}

\begin{abstract}
Objective: Histologic grade and Ki-67 proliferation status are important clinical indictors for breast cancer prognosis and treatment. The purpose of this study is to improve prediction accuracy of these clinical indicators based on tumor radiomic analysis. Methods: We jointly predicted $\mathrm{Ki}-67$ and tumor grade with a multitask learning framework by separately utilizing radiomics from tumor MRI series. Additionally, we showed how multitask learning models (MTLs) could be extended to combined radiomics from the MRI series for a better prediction based on the assumption that features from different sources of images share common patterns while providing complementary information. Tumor radiomic analysis was performed with morphological, statistical and textural features extracted on the DWI and dynamic contrast-enhanced MRI (DCE-MRI) series of the precontrast and subtraction images, respectively. Results: Joint prediction of Ki-67 status and tumor grade on MR images using the MTL achieved performance improvements over that of single-task-based predictive models. Similarly, for the prediction tasks of Ki-67 and tumor grade, the MTL for combined precontrast and apparent diffusion coefficient (ADC) images achieved AUCs of 0.811 and 0.816 , which were significantly better than that of the single-taskbased model with $p$ values of 0.005 and 0.017 , respectively. Conclusion: Mapping MRI radiomics to two related clinical indicators improves prediction performance for both Ki-67 expression level and tumor grade. Significance: Joint prediction of indicators by multitask learning that combines correlations of MRI radiomics is important for optimal tumor therapy and treatment because clinical decisions are made by integrating multiple clinical indicators.
\end{abstract}

Index Terms - Breast cancer, Ki-67, histologic grade, radiomics, multitask learning

\section{INTRODUCTION}

$\mathrm{B}$ REAST cancer pathological grade and Ki-67 expression level are important clinical indicators for breast cancer diagnosis and treatment. Tumor histological grade, a wellestablished prognostic factor, is crucial for guiding the appropriate treatment in clinical practice [1]. The Ki-67 index is an indicator of tumor aggressiveness and proliferative activity [2], in which a higher expression level reliably indicates

This work has received funding by the National Natural Science Foundation of China $(61731008,61871428$, and 61401131), the Natural Science Foundation of Zhejiang Province of China (LJ19H180001), and by the King Abdullah University of Science and Technology (KAUST) Office of Sponsored Research (OSR) under Award No. URF/1/3450-01.

M. Fan, W. Zhao and W. Yuan are with College of Life Information and Instrument Engineering, Hangzhou Dianzi University. more aggressive growth of breast cancer [3] and often a poorer pathological complete response (pCR) [3-7]. A large metaanalysis indicates that higher $\mathrm{Ki}-67$ expression is associated with a greater risk of recurrence [8] and poorer prognosis [9].

At the time of diagnosis, imaging is primarily used to establish the clinical stage before treatment intervention to drive the decision making. Dynamic contrast-enhanced magnetic resonance imaging (DCE-MRI) is a highly sensitivity method for diagnosing invasive breast cancer, whereas the specificity is only moderate [10]. Diffusion-weighted imaging (DWI), on the other hand, is performed without the administration of contrast agents, and it measures the Brownian motion of free water in tissue that is affected by fluid viscosity, membrane permeability, fibers, and tissue cellularity by using a quantitative method to assess the apparent diffusion coefficient (ADC). This technology is increasingly used because it can add the specificity of breast tumor diagnosis and is complementary to DCE-MRI for tumor characterization $[11,12]$.

However, to predict tumor characteristics, only a single parametric MRI is usually used. For example, studies indicate that radiomic analysis of MRI may be helpful in predicting breast cancer subtypes [13-16], neoadjuvant chemotherapy responses [17-19], Ki-67 expression level [20-22], pathological stage [23], histological grade [24], tumor malignancy [25, 26], and breast cancer risk [27] based on either DCE-MRI or DWI. These studies were performed without considering complementary information that could be provided by different parametric images.

Great efforts have been devoted in utilizing MRI [28-32], including DCE-MRI, T2-weighted imaging or DWI for the diagnosis and treatment of breast cancer. For example, Partridge et al. improved the diagnostic accuracy of breast MRI through combined ADC and dynamic kinetics. Shin et al. extracted MRI and DWI features separately to predict the Ki67 expression level. However, these studies were performed using separate images or by simply averaging the predictions without considering the common and complementary information of the image features.

On the other hand, although studies have shown the power of associating tumor radiomics and genomic or clinical features,

S. Wang and M. Xu are with Department of Radiology, First Affiliated Hospital of Zhejiang Chinese Medical University, Hangzhou, China.

X. Gao is with King Abdullah University of Science \& Technology, Thuwal, Saudi Arabia.

L. Li is with College of Life Information and Instrument Engineering, Hangzhou Dianzi University, Hangzhou 310018, China. (e-mail: lilh@hdu.edu.cn) 
most existing studies focus on separately estimating multiple clinical variables $[24,33,34]$ and thus cannot utilize the intrinsically useful, correlated information among these variables. Different biomarkers provide complementary information for cancer treatment and diagnosis. Their measurements capture common histopathological information by jointly characterizing a patient's tumor status. For example, patients with breast cancer who show high Ki-67 expression levels are associated with high histological grade [35]. Importantly, while each clinical indicator gives limited and noisy information, considering them jointly provides a more complete and comprehensive diagnostic depiction, as diagnosis is usually based on a combination of these assessments. In the machine learning community, the joint estimations of Ki-67 expression level and tumor grade in breast cancer can be formulated as a multitask classification problem [36]. Multitask learning methods have been applied in brain images to jointly predict behavioral scores that make up the individual profiles [37], and the results of improved predictions justify the idea.

However, whether the prediction accuracy of correlating between combined MRI radiomics and multiple clinical variables is better than that of associating single parametric MR radiomics with one clinical indicator is still an open question. Motivated by the above analysis, we aim to discriminate the histological grade and $\mathrm{Ki}-67$ expression status jointly by multitask feature learning and classification to improve the prediction accuracy. We also aim to examine whether DCEMRI and DWI can improve the prediction performance compared with that based on a single parametric image. Specifically, our contributions are threefold.

First, we established a multitask radiomic classification model for simultaneously discriminating Ki-67 and tumor grade in breast cancer. To the best of our knowledge, this is the first study to take advantage of tumor radiomics based on multitask feature selection (MTFS) and classification methods for the prediction of multiple clinical indicators. Our method can reduce possible noise in individual measurements of tumors and may be promising for improving the classifier performance of both targets. Second, our proposed model is flexible and extendible because more image series, such as $\mathrm{T} 2$-weighted images, and more clinical indicators, such as molecular subtypes (e.g., luminal A and liminal B), could be incorporated in the multitask learning-based radiomic analysis. Finally, the proposed model is important for optimal tumor therapy and treatment because clinical decisions are made by integrating multiple clinical indicators obtained from tumor biopsy.

\section{MAterials AND MethodS}

\section{A. Dataset}

We initially collected 203 biopsy samples indicating invasive breast cancer from the First Affiliated Hospital of Zhejiang Chinese Medical University between March 2013 and May 2017 (Fig. 1). The institutional review boards (IRB) approved this study (No. 2018001), and the requirement for informed consent was waived due to the retrospective nature of this study. Twenty-four of the patients who had incomplete MR sequence data and three for whom there was no pathological report available were excluded from the dataset. Thirty-two patients were excluded because there was no histological grading information in their pathological reports. Thus, the final dataset included 144 women with a mean age of 52 years and an age range of 28-83 years. There were 68 low-grade (grade 2) and 76 high-grade (grade 3) tumors and 36 low Ki-67 and 108 high $\mathrm{Ki}-67$ expression samples. There were no grade 1 tumors in our dataset because the number of this tumor grade is quite small in the initial collection of the data cohort (i.e., only 3 samples) and were excluded due to noncompliance with the selection criteria (i.e., incomplete MR sequence).

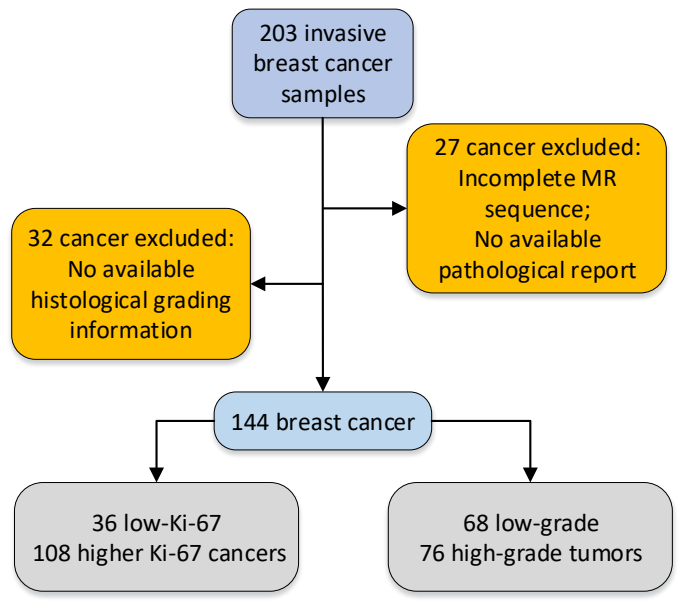

Fig. 1. Flowchart of patient selection.

All breast MR examinations were performed with a wide-bore Magnetom Verio 3.0T system (Siemens Medical Systems, Erlangen, Germany) fitted with a dedicated eight-channel breast array coil with the patient placed in the prone position. The DCE-MRI included one precontrast and five postcontrast bilateral sagittal image acquisitions performed with a fatsuppressed T1-weighted three-dimensional sequence. The following parameters were used: repetition time (TR) $4.51 \mathrm{~ms}$, echo time (TE) $1.61 \mathrm{~ms}$, matrix $448 \times 448$, flip angle $10^{\circ}$, field of view (FOV) $340 \times 340 \mathrm{~mm}$, slice thickness $1 \mathrm{~mm}$, and inplane resolution $0.759 \times 0.759 \mathrm{~mm}$. A bolus of $0.2 \mathrm{mmol} / \mathrm{kg}$ gadobutrol was intravenously injected at a rate of $2 \mathrm{~mL} / \mathrm{second}$ using an MR imaging-compatible power injector. The first postcontrast acquisition was performed 60 seconds after the contrast material was injected. Five sequential contrastenhanced images were then obtained with a temporal resolution of 60 seconds.

DWI was performed before the precontrast MR scan using the following parameters: $\mathrm{TR}=8400 \mathrm{~ms}$ (or $7000 \mathrm{~ms}$ ), $\mathrm{TE}=84$ $\mathrm{ms}$ (or $85 \mathrm{~ms}$ ), flip angle $=90^{\circ}, \mathrm{FOV}=147 \mathrm{~mm} \times 359 \mathrm{~mm}$ (or $120 \times 339$ ), slice thickness $=4.0 \mathrm{~mm}$, spacing between slices $=$ $6.0 \mathrm{~mm}$, and pixel bandwidth $=1263 \mathrm{~Hz}$ (or $1196 \mathrm{~Hz}$ ). The acquisition matrix was $220 \times 90$ with a spatial resolution of 1.64 $\mathrm{mm}$ and $\mathrm{b}$ values $=50,400$ and $800 \mathrm{~s} / \mathrm{mm}^{2}$. The ADC maps were obtained from diffusion images with three $b$ values.

Two pathologists with more than 15 years of experience who were blinded to the MRI results analyzed the breast tissue specimens. The histological type of breast cancer was defined according to the WHO classification [38]. Tumor histological grade was assessed using the Elston-Ellis System. For Ki-67, cases with more than $14 \%$ positive nuclei were determined as high Ki-67 expression, and other tumors were considered lowKi-67 expression according to the St. Gallen consensus [39]. 


\section{B. Image Processing}

The location of the center of a suspicious breast tumor was first annotated in each case retrospectively via consensus between two radiologists (S.W. and M.X.) who have more than 10 years of experience. Image segmentation was performed on the third postcontrast series for all cases because this series is usually the most enhanced of all the time series and hence is usually useful for visual inspection. An initial "seed" was chosen using the location of the center of the labeled tumor. After that, a volumetric breast tumor boundary contour was automatically segmented by a spatial fuzzy C-means (FCM) algorithm and subsequently refined by a Markov random field (MRF)-based approach, the parameters of which were adaptively adjusted using the segmentation results of contiguous slices [40]. In less than $10 \%$ of all cases, the segmentation results were further examined by manual correction performed by our investigators. The breast MR images acquired from sequential MRI scans were registered as previously reported [40].

\section{Radiomic Features}

The radiomic features were derived from the precontrast images (S0), and from the subtraction images between the second postcontrast or the fifth postcontrast images and the precontrast images; these subtraction images were termed S20, and S50, respectively. Additionally, the tumor region of interest (ROI) on the DCE-MRI was aligned to that of the ADC map using the Statistical Parametric Modeling toolbox version 12 (SPM12) platform (http://www.fil.ion.ucl.ac.uk/spm/software/spm12). Imaging Features, including textural, statistical and morphological features were extracted from the DCE-MRI and ADC maps. To quantify spatial heterogeneity within each tumor, we extracted 19 volumetric textual features that was calculated from gray-level cooccurrence matrices (GLCM) [4143]. To reduce the feature space dimension, texture features are extracted from the nondirectional GLCMs by summing up the 13 directional GLCMs, which is a common practice reported in the literature [44].

Although the gray-level quantization could affect the calculated texture features, different groups tend to use arbitrarily chosen quantization methods when constructing the GLCM [44]. A previous study analyzed quantization parameters of texture, and recommend the same number of gray levels in all quantized images for texture features [45]. Following the previous studies, we used a fixed number of 32 bits for texture feature analysis [46].

We obtained 10 statistical features for evaluating the histogram distributions of image pixels inside the tumor. Additionally, we calculated 10 morphological features to measure the shape of the tumor. The detailed image features are listed in TABLE I. The textural and statistical features were extracted from all the image series, whereas the morphological features were computed only from the S0 image and ADC map. Therefore, 97 features were calculated for each tumor.

\section{Multitask Learning Methods}

We wanted to simultaneously improve the prediction of pathological grading and $\mathrm{Ki}-67$ proliferation status from the DCE-MRI and ADC images. Fig. 2 depicts the workflow of the proposed model, which is a multitask learning model built with a two-layer architecture. The first step performs a supervised MTFS on each image series separately and yields a feature set that is correlated to both the Ki-67 level and the tumor grade for each image series. The second level learns a common multioutput model using the generated feature sets in MTFS and returns the final prediction of the clinical indicators based on a combination of all the image series.

Feature selection is used for dimensionality reduction, as well as for removal of irrelevant features derived from MR images.

TABLE I

FEATURE DESCRIPTION

\begin{tabular}{|c|c|}
\hline Feature & Description \\
\hline \multicolumn{2}{|l|}{ Texture feature } \\
\hline Autocorrelation & Detect repetitive patterns of texture elements \\
\hline Cluster prominence & Asymmetry \\
\hline Cluster shade & Asymmetry or uniformity \\
\hline Contrast & Local variations presented in an image \\
\hline Correlation & Image complexity \\
\hline Difference entropy & $\begin{array}{l}\text { Randomness of the difference in neighboring } \\
\text { voxel gray levels }\end{array}$ \\
\hline Difference variance & Variations of difference in gray level pairs \\
\hline Dissimilarity & Local contrasts \\
\hline Energy & Uniformity of the image gray distribution \\
\hline Entropy & Randomness of the intensity distribution \\
\hline Homogeneity & Uniformness of pixel pair \\
\hline Information correlation & Nonlinear gray level dependence \\
\hline Information correlation & Nonlinear gray level dependence \\
\hline Inverse difference & Local similarity \\
\hline Maximum probability & Highest frequency of pixel pair \\
\hline Sum average & Overall brightness \\
\hline Sum entropy & $\begin{array}{l}\text { Randomness of the sum of gray levels of } \\
\text { neighboring voxels }\end{array}$ \\
\hline Sum of squares variance & Spread in the gray level distribution \\
\hline Sum variance & $\begin{array}{l}\text { Spread in the sum of the gray levels of voxel- } \\
\text { pairs distribution }\end{array}$ \\
\hline \multicolumn{2}{|l|}{ Statistical feature } \\
\hline Entropy & Randomness of the intensity distribution \\
\hline Interquartile range & A robust estimate of the spread of the data \\
\hline Kurtosis & Distribution apart from the variance \\
\hline Maximum & The maximum pixel gray value of the lesion \\
\hline Mean & The average pixel gray value of the lesion \\
\hline Median & The median pixel gray value of the lesion \\
\hline Minimum & The minimum pixel gray value of the lesion \\
\hline Range & An indication of statistical dispersion \\
\hline Skewness & Asymmetry of the histogram \\
\hline Standard deviation & $\begin{array}{l}\text { Variation in or dispersion of pixel value of } \\
\text { the lesion }\end{array}$ \\
\hline \multicolumn{2}{|l|}{ Morphological feature } \\
\hline Compactness & The degree to which the lesion is compact \\
\hline Compactness 1 & The degree to which the lesion is compact \\
\hline Compactness 2 & The degree to which the lesion is compact \\
\hline Eccentricity & $\begin{array}{l}\text { Covariance between morphological shape } \\
\text { elements }\end{array}$ \\
\hline Equivalent radius & $\begin{array}{l}\text { Radius of a sphere with the same volume as } \\
\text { the lesion }\end{array}$ \\
\hline Max radius & The maximum radius of the lesion sections \\
\hline Mean radius & The mean radius of the lesion sections \\
\hline Surface area & Surface area of the lesion \\
\hline Surface area/volume & Ratio of the surface area to volume \\
\hline Volume & Volume of the lesion \\
\hline
\end{tabular}




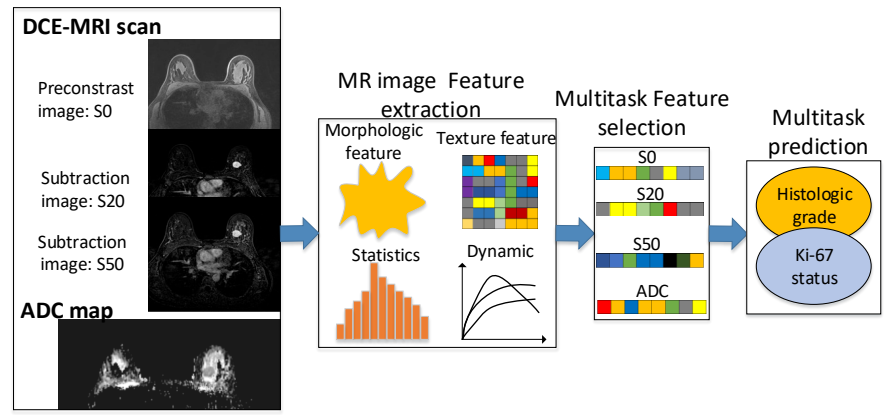

Fig. 2. Overview of the proposed method. Multitask learning for radiomic analysis of DCE-MRI and ADC map.

In contrast to conventional single-task feature selection, MTFS simultaneously selects a common feature subset relevant to all tasks. This point is especially important for breast cancers because the clinical indicators are determined by the same underlying cancer pathology of the tumor. In addition, simultaneously performing the feature selection for clinical variables such as Ki-67 and histological grade can be very helpful for suppressing noise in the individual variables. We used multitask elastic-net regression, which can enforce sparse restrictions for a given regularization, assuming that different tasks share the same parameter that control sparsity. Assuming there are $T$ tasks and $M$ image series, the $i^{\text {th }}$ images with an input matrix $X_{i} \in R^{N \times V}$ denote the input matrix for $N$ subjects and $V$ features, and the output matrix $Y \in R^{N \times T}$ denotes the labels of $N$ subjects for $T$ tasks. The coefficient matrix $W_{i} \in R^{V \times T}$ for the $i^{\text {th }}$ image is estimated as follows:

$$
\widehat{W}_{i}=\underset{W_{i} \in R^{V \times T}}{\operatorname{argmin}}\left\|Y-X_{i} W_{i}\right\|_{F}^{2}+a\left\|W_{i}\right\|_{1}+\beta\left\|W_{i}\right\|_{F}^{2}
$$

where the regularization parameter $a>0$ controls the sparsity of the estimate, and the parameter $\beta$ controls the $\ell_{2}$-norm penalty. $\left\|W_{i}\right\|_{F}^{2}$ is the Frobenius norm of matrix $W_{i}$, which can be computed by a trace of $W_{i}{ }^{T} W_{i}$, and $\beta$ is the regularization parameter that controls the amount of shrinkage, i.e., the larger the value of $\beta$ is, the greater the amount of shrinkage. In this elastic-net, both the $\ell_{1}$-norm and $\ell_{2}$-norm are used. If a feature is not relevant across all tasks, the corresponding row in the estimated matrix $\widehat{W}_{i}$ is zero. After the MTFS procedure, the estimated weight matrix $W$ is used to define the selected features for its nonzero entries to perform multitask prediction optimally. At present, many algorithms have been developed to solve MTFS; in this paper, we adopt the MALSAR-toolbox [47].

In this study, we developed a multiple images-based classifier with features selected by the above MTFS method. After obtaining the common feature subset for all different tasks by MTFS as described above, we used a multitask learning method to train the final classification models. We utilized a multitask classifier (MTC) in which the matrix that models the relations between tasks is learned using a convex optimization problem that can be approached by means of alternating minimization [48]. Prediction from each image was integrated by an average method weighted by the accuracy of the trained model in a cross-validation procedure.

\section{E. Statistical Analysis}

At the preprocessing stage, we performed a common feature normalization step, i.e., subtracting the mean and then dividing the standard deviation (of all training subjects) for each feature value. Univariate logistic regression analysis was performed to assess the predictive power of individual predictors. We used a nested leave-one-out cross-validation (LOOCV) strategy in the training and classification tasks. Specifically, in each LOOCV loop, one subject sample was selected as the testing sample, and the remaining subjects were used for training the predictive models, in which the elastic-net parameters (i.e., $\alpha$ and $\beta$ ) were re-optimized by the grid search method. This process was repeated $N$ times, each of which generated a predictive variable to produce a prediction performance, i.e., an area under the receiver operating characteristic (ROC) curve (AUC) value. The significance of the comparison between the AUC values was assessed using a bootstrapping test (pROC package in R program). Sensitivity and specificity were obtained at maximum Youden's index derived from ROC curves. All $\mathrm{p}$ values were corrected for false discovery (FDR) correction using the Benjamini-Hochberg method. Statistical analysis was performed using MATLAB (R2015) and R (version 3.4.1).

\section{RESULTS}

\section{A. Patients}

Table II shows the histopathological characteristics of the 144 patients. The statistical tests showed a significant association between histological grade and $\mathrm{Ki}-67$ expression level $(\mathrm{p}=0.001)$. No significant differences in histological information, including progesterone receptor (PR) status, menopausal status, age, and $\mathrm{Ki}-67$ status, were observed between the low and high Ki-67 expression breast tumors. We observed significant differences in the maximum tumor diameter between the low and high Ki-67 expression tumors.

TABLE II

TUMOR CHARACTERISTICS

\begin{tabular}{|c|c|c|c|c|}
\hline & $\begin{array}{l}\text { All } \\
144\end{array}$ & $\begin{array}{l}\text { Low-Ki- } \\
67 \\
(\mathrm{n}=36) \\
(\%) \\
\end{array}$ & $\begin{array}{l}\text { High-Ki-67 } \\
(\mathrm{n}=108) \\
(\%)\end{array}$ & $\begin{array}{l}\mathrm{P} \\
\text { value* }\end{array}$ \\
\hline $\mathrm{PR}^{\pi}$ & & & & 0.078 \\
\hline Positive & 90 & $27(18.75)$ & $63(43.75)$ & \\
\hline Negative & 54 & $9(6.25)$ & $45(31.25)$ & \\
\hline Histological grade & & & & 0.001 \\
\hline 2 & 68 & $26(18.1)$ & $42(29.2)$ & \\
\hline 3 & 76 & $10(6.9)$ & $66(45.8)$ & \\
\hline Menopausal status & & & & 0.440 \\
\hline Premenopausal & 78 & $22(15.3)$ & $56(38.9)$ & \\
\hline Postmenopausal & 66 & $14(9.7)$ & $52(36.1)$ & \\
\hline $\operatorname{Age}(y)^{\ddagger}$ & $\begin{array}{ll}52.1 \quad(28- \\
83)\end{array}$ & $\begin{array}{l}51.3(28- \\
83) \\
\end{array}$ & $\begin{array}{l}52.42(31- \\
82) \\
\end{array}$ & 0.605 \\
\hline $\begin{array}{ll}\text { Maximum } & \text { tumor } \\
\text { diameter }(\mathrm{mm})^{\ddagger} & \\
\end{array}$ & $\begin{array}{l}23.44(6- \\
100) \\
\end{array}$ & $\begin{array}{l}17.61(6- \\
50)\end{array}$ & $\begin{array}{l}25.4(7- \\
100) \\
\end{array}$ & $<0.001$ \\
\hline
\end{tabular}

"P value for low Ki-67 versus high Ki-67 comparison.

'The data were tested using Fisher's exact test.

${ }^{\dagger}$ The data were tested using a t-test.

$\mathrm{PR}$, progesterone receptor; numbers in parentheses are percentages

Tumors with $\mathrm{Ki}-67>14 \%$ were considered high-Ki-67; others were considered low-Ki-67

\section{B. Univariate Analysis of Image Features}

The ability of individual features to predict tumor grade and Ki67 expression status is illustrated in Fig. 3 and Supplementary Fig. 1, respectively. The results show that textural and morphological features have relatively greater AUC values that are significantly (corrected $\mathrm{p}$ values $<0.05$ ) higher than random 


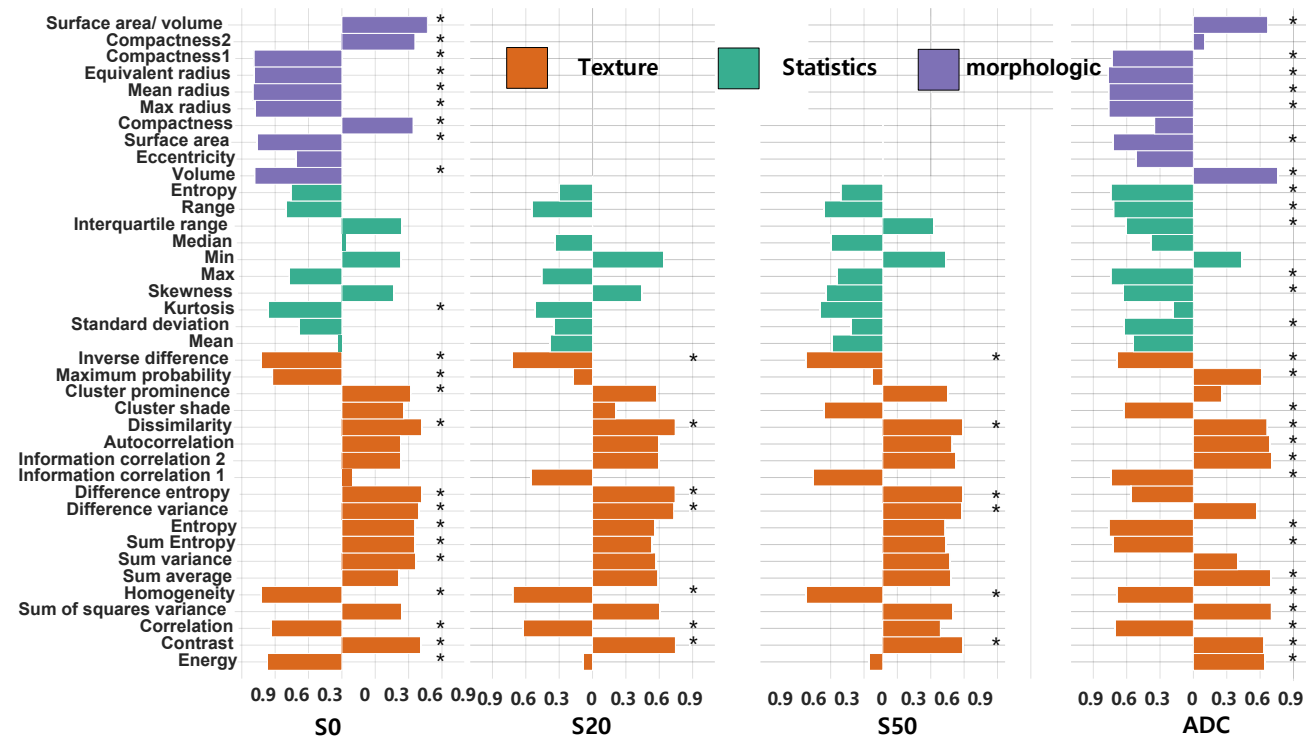

Fig. 3. Individual AUC values of texture, statistic, and shape features from the images for prediction of histological grade. The predictive power is reported as directly or inversely proportional to the tumor grade as the feature value increased, which is represented on the right and left sides of the bar diagram,

respectively. Features reported with an asterisk are significant from a random guess (Bootstrap test, $\mathrm{p}<0.05$, false discovery rate-corrected).

values (i.e., $\mathrm{AUC}=0.5$ ). However, no statistical features in the MR images (i.e., S0, S20, S50 and ADC) showed high predictive power (corrected $\mathrm{p}$ values $>0.05$ ). As illustrated in Fig. 4, the texture feature of difference entropy showed a significantly higher $(\mathrm{p}$ value $<0.001)$ value in the low Ki-67 expression tumors for image S0, S20 and S50. This significant, high trend was also observed for low histological grade tumors compared with that for high-grade tumors ( $\mathrm{p}$ values $<0.001)$. On the other hand, we observed a different trend in this feature for comparing between the two groups in ADCs, in which a significantly higher value of this feature was found in high Ki67 or grade 3 tumors than in low Ki-67 or grade 2 tumors.

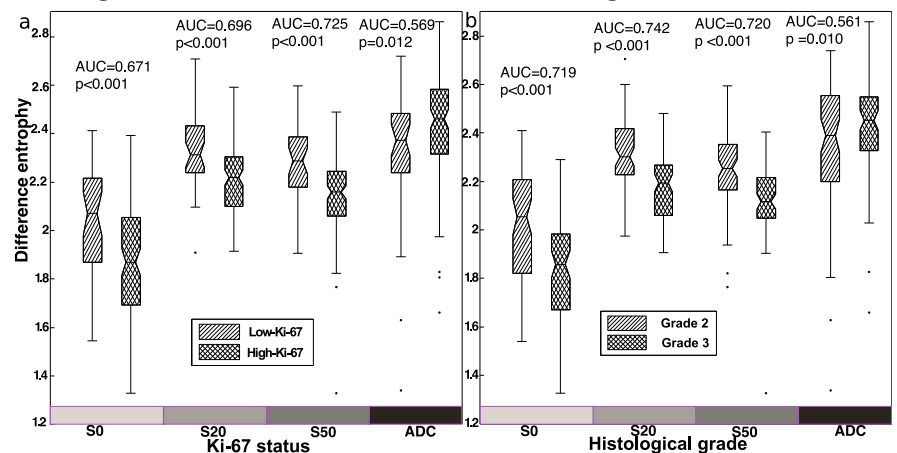

Fig. 4. Distribution of DCE-MRI and ADC texture features of difference entropy for prediction of a) $\mathrm{Ki}-67$ status and b) histological grade in breast cancer patients.

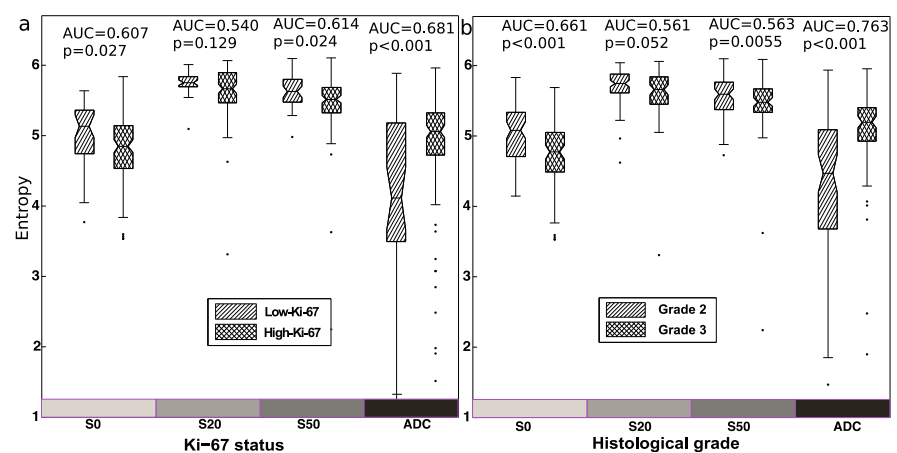

Fig. 5. Distribution of DCE-MRI and ADC texture features of entropy for prediction of a) Ki-67 status and b) histological grade in breast cancer patients.
We also observed that entropy features based on ADC had high discriminative power in the histological prediction task $(\mathrm{AUC}=0.763$ and $\mathrm{p}$ value $<0.001)$ and moderate predictive ability for predicting $\mathrm{Ki}-67$ status (AUC $=0.681$ and $p$ value $<$ 0.001 ), which were among the best individual performance features (Fig. 5). However, for the DCE-MRI series (i.e., S0, S20, and S50), entropy features showed a less significant association with either Ki-67 or histological grade in breast cancer. More specifically, a higher value of this feature was observed in low Ki-67 and low histological grade tumors than in high Ki-67 or high-grade tumors for the DCE-MRI series, a result that was opposite to that based on the ADC images.

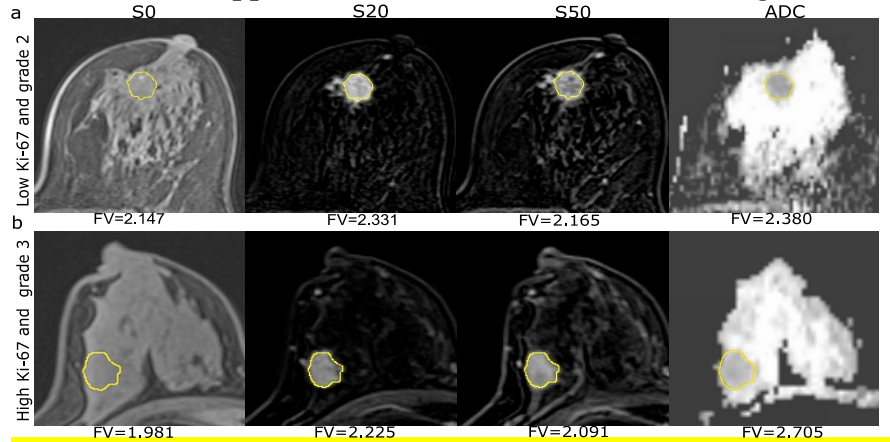

Fig. 6. Examples of image feature values of difference entropy for a) tumor with low Ki-67 expression level and histological grade 2 and b) tumor with high Ki67 expression level and histological grade 3 . Feature values are derived from images of S0, S20, S50 and ADC, respectively. FV=feature value.

Fig. 6 illustrates an example of a difference entropy feature in a low Ki-67 and low-grade patient compared with that in a patient with a high Ki-67 and high-grade tumor from the images of S0, S20, S50 and ADC. Patients with low Ki-67 and lowgrade tumors showed higher values of difference entropy than did those with high Ki-67 and high-grade tumors. On the other hand, for ADC, this feature showed an inverse trend between the two groups.

\section{Single-Task Prediction Using Features from ADC and DCE-MRI Series Separately}

We separately examined the performance of single-task methods for predicting Ki-67 expression or histological grade 
TABLE III

Classifier PeRformances For TUMOR Ki-67 Status AND Histological GRAde

\begin{tabular}{lllcccccc}
\hline \hline \multirow{2}{*}{ Predictive model } & $\begin{array}{l}\text { Feature } \\
\text { selection }\end{array}$ & \multirow{2}{*}{ Images } & \multicolumn{3}{c}{ Ki-67 } & \multicolumn{3}{c}{ Histological grade } \\
\cline { 3 - 8 } Logistic & Lasso & S0 & $0.756 \pm 0.086$ & 0.731 & 0.722 & $0.780 \pm 0.078$ & 0.947 & 0.574 \\
regression & & S20 & $0.737 \pm 0.101$ & 0.713 & 0.722 & $0.766 \pm 0.081$ & 0.947 & 0.529 \\
& & S50 & $0.733 \pm 0.093$ & 0.676 & 0.694 & $0.765 \pm 0.082$ & 0.934 & 0.544 \\
& & ADC & $0.738 \pm 0.089$ & 0.501 & 0.917 & $0.757 \pm 0.082$ & 0.737 & 0.721 \\
Logistic & RFE & S0 & $0.778 \pm 0.084$ & 0.648 & 0.806 & $0.796 \pm 0.074$ & 0.803 & 0.691 \\
regression & & S20 & $0.766 \pm 0.097$ & 0.759 & 0.750 & $0.774 \pm 0.081$ & 0.961 & 0.618 \\
& & S50 & $0.757 \pm 0.093$ & 0.787 & 0.667 & $0.743 \pm 0.084$ & 0.816 & 0.618 \\
& & ADC & $0.750 \pm 0.089$ & 0.593 & 0.889 & $0.742 \pm 0.085$ & 0.895 & 0.559 \\
SVM & RFE & S0 & $0.664 \pm 0.113$ & 0.852 & 0.556 & $0.741 \pm 0.089$ & 0.908 & 0.588 \\
& & S20 & $0.660 \pm 0.121$ & 0.815 & 0.556 & $0.754 \pm 0.085$ & 0.842 & 0.662 \\
& & S50 & $0.584 \pm 0.118$ & 0.602 & 0.639 & $0.756 \pm 0.081$ & 0.697 & 0.735 \\
MTC & ADC & $0.573 \pm 0.117$ & 0.796 & 0.444 & $0.757 \pm 0.085$ & 0.789 & 0.691 \\
& Lasso & S0 & $0.761 \pm 0.087$ & 0.574 & 0.889 & $0.786 \pm 0.078$ & 0.961 & 0.559 \\
& & S20 & $0.738 \pm 0.098$ & 0.731 & 0.694 & $0.768 \pm 0.079$ & 0.987 & 0.471 \\
& & S50 & $0.775 \pm 0.086$ & 0.787 & 0.667 & $0.788 \pm 0.077$ & 0.776 & 0.721 \\
& & ADC & $0.771 \pm 0.083$ & 0.537 & 0.917 & $0.785 \pm 0.076$ & 0.895 & 0.588 \\
\hline \hline
\end{tabular}

$\overline{\mathrm{RFE}}=$ recursive feature elimination, LASSO = least absolute shrinkage and selection operator, SVM = support vector machine.

based on features from the ADC and DCE-MRI series. We tested the classifiers of least absolute shrinkage and selection operator (lasso), logistic regression, support vector machine (SVM), and MTC [48] for prediction. The performance of AUC values is listed in Table III.

The result shows that the S20-based predictive model had lower performance than did the models based on the other images. For Ki-67 status, the predictive model used features derived from S0, S20, S50 and ADC images with recursive feature elimination (RFE) as the feature selection and logistic regression as the classifier [48] and had AUCs of 0.778, 0.766, 0.757 and 0.750 , respectively. For tumor grade prediction, the images of S0, S20, S50 and ADC showed relatively high prediction performance in terms of AUCs $(0.796,0.774,0.743$ and 0.742 , respectively). Although different classifiers were utilized, the performance for the prediction tasks was similar.

\section{Multitask Learning Improves Single Parametric Image- based Prediction Performance}

We applied multitask learning for single-model prediction on the DCE (S0, S20, and S50) and ADC images separately. MTFS was conducted using the least squares loss. The ROC curves of S20 in single and multitask learning for predicting $\mathrm{Ki}-67$ and tumor grade are shown in Fig. 7. The selected features that commonly target the two indicators were then used in the multitask learning classifier. The prediction of the two tasks was performed using a multitask classifier [48]. The results showed that prediction performance was increased for all image series, while MTFS and classification methods were utilized (Table IV and Fig. 8). The performance of the S20-based predictive model was significantly improved by using multitask learning both for the Ki-67 task and tumor grade in breast cancer, with $p$ values of 0.032 and 0.024 , respectively.

We tested the proposed framework by comparing the prediction performance of multitask learning to those of singletask for the MR images. For the Ki-67 prediction, multitask learning using images of S20, S50 and ADC significantly ( $p<$ 0.05 ) improved the single-task based prediction performance

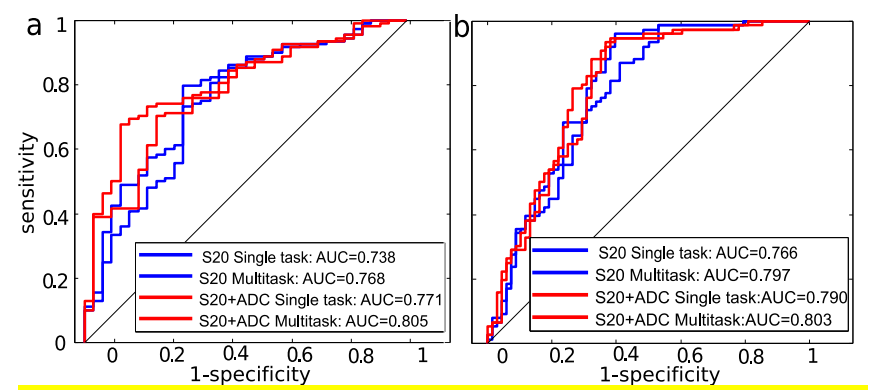

Fig. 7. ROC of the single-task and multitask learning on the S20 and the combination with $\mathrm{ADC}$ series.

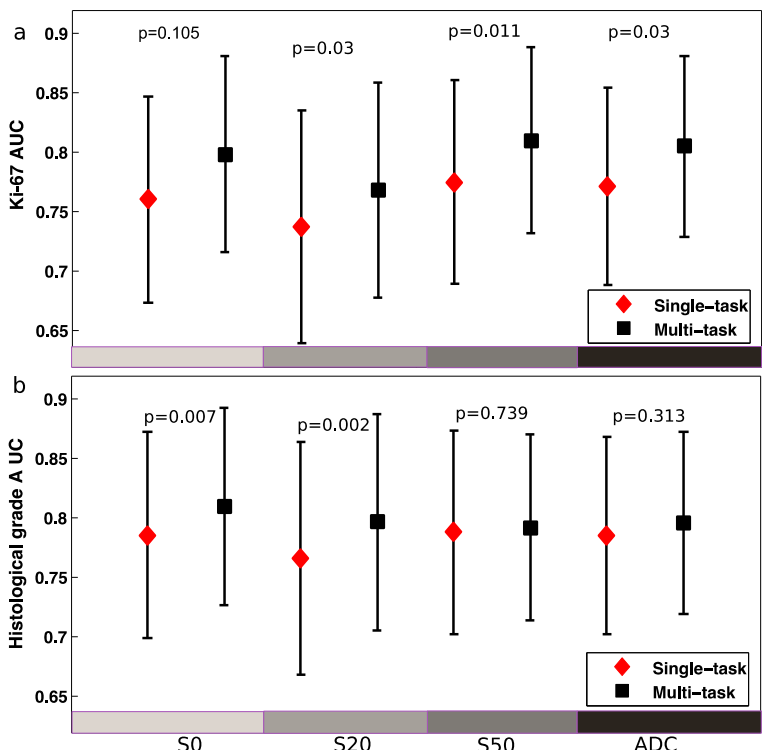

Fig. 8. Multitask learning improves classifier performance for both the a) Ki67 and b) histological grade prediction tasks.

(in terms of AUCs). For histological grade prediction, multitask learning using images of S0 and S20 significantly $(\mathrm{p}<0.05)$ improved predictive model performance. 
TABLE IV

MULTITASK LEARNING PERFORMANCES USING DCE-MRI AND ADC IMAGES

\begin{tabular}{|c|c|c|c|c|c|c|c|}
\hline \multicolumn{8}{|c|}{ Ki-67 } \\
\hline \multirow{2}{*}{ Images } & \multicolumn{3}{|c|}{ Single-task } & \multicolumn{4}{|c|}{ Multitask } \\
\hline & AUC & Sens & Spec & AUC & Sens & Spec & $P$ value \\
\hline S0 & 0.761 & 0.574 & 0.889 & 0.795 & 0.769 & 0.750 & 0.105 \\
\hline S20 & 0.738 & 0.731 & 0.694 & 0.768 & 0.796 & 0.694 & 0.032 \\
\hline $\mathrm{S} 50$ & 0.775 & 0.787 & 0.667 & 0.810 & 0.843 & 0.667 & 0.011 \\
\hline $\mathrm{ADC}$ & 0.771 & 0.537 & 0.917 & 0.805 & 0.676 & 0.917 & 0.028 \\
\hline \multicolumn{8}{|c|}{ Histological Grade } \\
\hline S0 & 0.786 & 0.574 & 0.889 & 0.810 & 0.769 & 0.750 & 0.007 \\
\hline S20 & 0.766 & 0.731 & 0.694 & 0.797 & 0.796 & 0.694 & 0.024 \\
\hline S50 & 0.788 & 0.787 & 0.667 & 0.792 & 0.843 & 0.667 & 0.739 \\
\hline $\mathrm{ADC}$ & 0.785 & 0.537 & 0.917 & 0.796 & 0.676 & 0.917 & 0.312 \\
\hline
\end{tabular}

Sens $=$ Sensitivity; Spec $=$ Specificity.

\section{E. Multitask Learning Improves MRI-radiomics-based Prediction Performance}

We combined radiomics from the MRI series to improve the performance of a single parametric image-based model for both single-task-based Ki-67 expression and tumor grade prediction. Classifiers that incorporated image features from S0, S20, S50 and ADC were evaluated, and the results showed that the performance of combined images was improved while the ADC features were introduced to the model (Table V). Additionally, compared with that for a single image, the prediction accuracy was moderately improved by combing features from the DCEMRI scans (i.e., S0, S20 and S50).

We further performed multitask learning to improve the model performance using image features from the DCE-MRI and ADC. Table $\mathrm{V}$ shows the multitask performance of the classifiers based on combined predictions from $\mathrm{S} 0, \mathrm{~S} 20, \mathrm{~S} 50$ and ADC. The prediction performance of MR images based on a single-task was improved by multitask learning (Table V). Additionally, while the MTFS and classification methods were utilized, most of the MR-image-based prediction performance (in terms of AUC values) was significantly improved $(p<0.05$ ) for Ki-67 status except for the classifier using the combined $\mathrm{S} 0$ and S20 images. While multitask learning was performed, the predictive model by integrating the S0 series with ADC was significantly improved for the Ki-67 and tumor grade prediction tasks ( $\mathrm{p}=0.005$ and 0.017 , respectively).

\section{F. Comparison of the Prediction Performance of Image Series Within Single-Task and Multitask Learning}

We compared the AUCs of a subset of MR series (i.e., DCEMRI and ADC images) within single-task and multitask learning (Fig. 9). The predictive model for S50 has significantly better prediction performance than that for S20 for both the single-task and multitask Ki-67 prediction ( $\mathrm{p}$ values of 0.044 and 0.011 , respectively). Moreover, the model performance based on the combination of the S0, S20 and S50 series has a significantly higher AUC value than that based on S20 for both the single-task and multitask learning models ( $\mathrm{p}$ values of 0.016 and 0.011 , respectively). For tumor histological grade prediction, the combined radiomics from the three DCE-MRI series showed the best prediction performance in single-task learning and was significantly $(\mathrm{p}=0.018)$ higher than that of the S20-based predictive model. However, we did not observe significantly different AUC values within multitask learning for these imaging series.

TABLE V

mpMri-Based Prediction Performance Using Single-TASK AND MUltitask Learning SeParately

\begin{tabular}{|c|c|c|c|c|c|c|c|}
\hline \multirow{3}{*}{ mpMRI } & \multicolumn{7}{|c|}{$\mathrm{Ki}-67$} \\
\hline & \multicolumn{3}{|c|}{ Single task } & \multicolumn{3}{|c|}{ Multitask } & \multirow[b]{2}{*}{$\mathrm{p}$ value } \\
\hline & AUC & Sensitivity & Specificity & AUC & Sensitivity & Specificity & \\
\hline $\mathrm{S} 0+\mathrm{S} 20$ & $0.761 \pm 0.088$ & 0.778 & 0.694 & $0.788 \pm 0.083$ & 0.796 & 0.694 & 0.068 \\
\hline $\mathrm{S} 0+\mathrm{S} 50$ & $0.780 \pm 0.083$ & 0.713 & 0.750 & $0.809 \pm 0.077$ & 0.713 & 0.806 & 0.026 \\
\hline $\mathrm{S} 0+\mathrm{S} 20+\mathrm{S} 50$ & $0.772 \pm 0.087$ & 0.778 & 0.694 & $0.798 \pm 0.081$ & 0.806 & 0.694 & 0.023 \\
\hline $\mathrm{S} 0+\mathrm{ADC}$ & $0.776 \pm 0.082$ & 0.556 & 0.944 & $0.811 \pm 0.076$ & 0.676 & 0.917 & 0.005 \\
\hline $\mathrm{S} 20+\mathrm{ADC}$ & $0.771 \pm 0.087$ & 0.704 & 0.778 & $0.805 \pm 0.078$ & 0.676 & 0.889 & 0.013 \\
\hline $\mathrm{S} 50+\mathrm{ADC}$ & $0.796 \pm 0.080$ & 0.778 & 0.722 & $0.821 \pm 0.074$ & 0.741 & 0.833 & 0.017 \\
\hline $\mathrm{S} 0+\mathrm{S} 20+\mathrm{ADC}$ & $0.777 \pm 0.084$ & 0.713 & 0.778 & $0.805 \pm 0.078$ & 0.694 & 0.889 & 0.040 \\
\hline $\mathrm{S} 0+\mathrm{S} 50+\mathrm{ADC}$ & $0.794 \pm 0.080$ & 0.787 & 0.722 & $0.820 \pm 0.074$ & 0.722 & 0.833 & 0.015 \\
\hline \multirow[t]{2}{*}{$\mathrm{S} 0+\mathrm{S} 20+\mathrm{S} 50+\mathrm{ADC}$} & $0.782 \pm 0.084$ & 0.741 & 0.750 & $0.810 \pm 0.077$ & 0.704 & 0.833 & 0.019 \\
\hline & \multicolumn{7}{|c|}{ Histological Grade } \\
\hline \multirow[t]{2}{*}{ mpMRI } & \multicolumn{3}{|c|}{ Single task } & \multicolumn{3}{|c|}{ Multitask } & \\
\hline & AUC & Sensitivity & Specificity & AUC & Sensitivity & Specificity & $\mathrm{p}$ value \\
\hline $\mathrm{S} 0+\mathrm{S} 20$ & $0.790 \pm 0.077$ & 0.842 & 0.691 & $0.815 \pm 0.074$ & 0.947 & 0.647 & 0.014 \\
\hline $\mathrm{S} 0+\mathrm{S} 50$ & $0.780 \pm 0.076$ & 0.921 & 0.618 & $0.812 \pm 0.075$ & 0.921 & 0.647 & 0.124 \\
\hline $\mathrm{S} 0+\mathrm{S} 20+\mathrm{S} 50$ & $0.794 \pm 0.077$ & 0.868 & 0.662 & $0.810 \pm 0.075$ & 0.908 & 0.662 & 0.071 \\
\hline $\mathrm{S} 0+\mathrm{ADC}$ & $0.798 \pm 0.075$ & 0.934 & 0.588 & $0.816 \pm 0.072$ & 0.803 & 0.750 & 0.017 \\
\hline $\mathrm{S} 20+\mathrm{ADC}$ & $0.790 \pm 0.077$ & 0.947 & 0.618 & $0.803 \pm 0.075$ & 0.934 & 0.632 & 0.141 \\
\hline $\mathrm{S} 50+\mathrm{ADC}$ & $0.799 \pm 0.076$ & 0.789 & 0.735 & $0.802 \pm 0.076$ & 0.921 & 0.618 & 0.709 \\
\hline $\mathrm{S} 0+\mathrm{S} 20+\mathrm{ADC}$ & $0.799 \pm 0.075$ & 0.882 & 0.676 & $0.816 \pm 0.073$ & 0.947 & 0.647 & 0.040 \\
\hline $\mathrm{S} 0+\mathrm{S} 50+\mathrm{ADC}$ & $0.808 \pm 0.074$ & 0.855 & 0.691 & $0.813 \pm 0.074$ & 0.961 & 0.618 & 0.506 \\
\hline $\mathrm{S} 0+\mathrm{S} 20+\mathrm{S} 50+\mathrm{ADC}$ & $0.802 \pm 0.076$ & 0.816 & 0.721 & $0.814 \pm 0.074$ & 0.947 & 0.662 & 0.119 \\
\hline
\end{tabular}




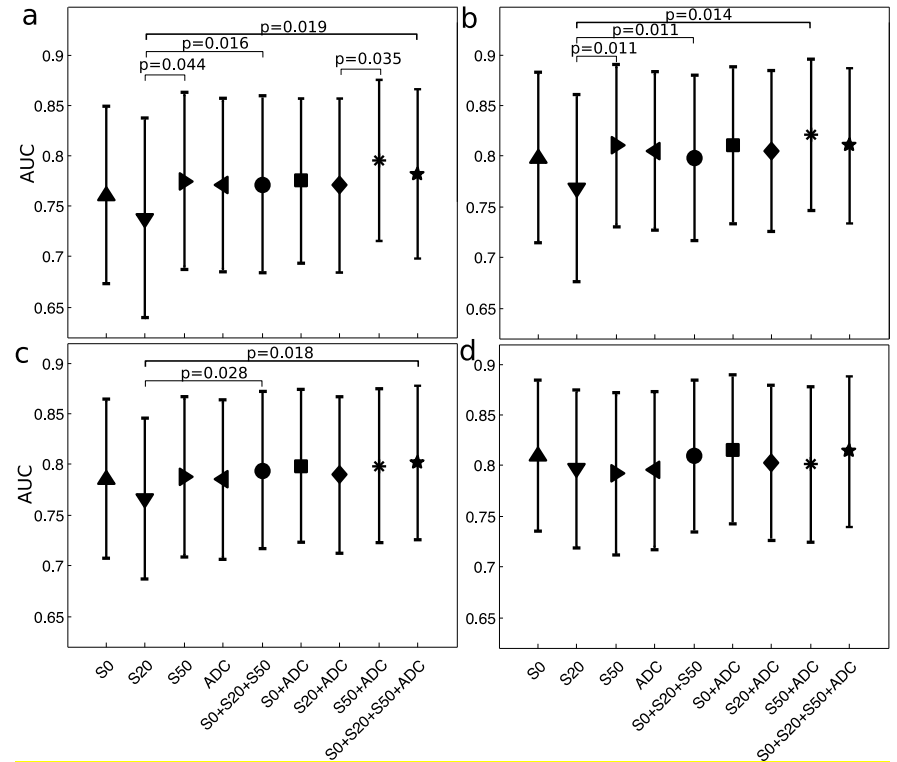

Fig. 9. Comparisons of AUCs within single-task and multitask learning for the MRI series. The prediction of $\mathrm{Ki}-67$ in a) single-task and b) multitask learning and that of tumor histological grade in c) single-task and d) multitask learning are shown.

\section{G. Case Study for Feature Selection in Single-task and Multitask Learning}

To evaluate and compare the feature selection frequency in single-task and multitask learning, we performed a case study for a S20-based predictive model targeting Ki-67 and tumor grade classification. The frequencies of selected features in the nested LOOCV method for these methods are shown in Table VI. Two morphological features (i.e., the ratio between surface area and volume and compactness 2 ) and one texture feature (i.e., information correlation 2) were mostly frequently selected in the cross-validation both for the Ki-67 and histological grade prediction tasks. The features of energy, homogeneity and volume were not included in the $\mathrm{Ki}-67$ prediction task but were selected with a frequency of more than $50 \%$ in multitask learning. Although the feature of mean radius has a relatively high AUC in the single-task (i.e., AUC value of 0.78 and 0.80 for the prediction of Ki-67 and tumor grade, respectively), it was less selected (41\%) in the multivariate model for the singletask prediction of histological grade, whereas it was most frequently selected $(100 \%)$ in multitask prediction. On the other hand, the feature of information correlation 1 was selected at approximately $50 \%$ in both the single-task of $\mathrm{Ki}-67$ and the histological grade, whereas was seldomly selected in multitask learning. This feature showed low prediction power in both the $\mathrm{Ki}-67$ (AUC=0.588) and tumor grade prediction tasks $(\mathrm{AUC}=0.555)$ and was almost never selected (i.e., $1.4 \%$ and $0 \%$ for the Ki-67 and histological grade tasks, respectively) in multitask learning.

\section{Discussion}

In this study, multitask learning was performed for the first time to predict $\mathrm{Ki}-67$ status and tumor grade in breast cancer specimens. Our study provides a promising attempt to predict multiple clinical indicators simultaneously following a normal diagnostic procedure. Multitask prediction models using tumor radiomics were designed, and the results suggested enhanced prediction accuracies for both tasks. Moreover, radiomics from DCE-MRI and DWI were combined and showed higher performance than that of the single parametric image.

The image features in this study have been reported by previous studies. For example, the feature of difference entropy, which measures heterogeneous textural appearance, i.e., randomness of the difference in neighboring voxel gray levels, was high in low Ki-67 expression tumors [49]. Moreover, a related study found that luminal A tumors had a higher value of this feature than luminal B tumors did [20]. Our results are partly consistent with those of the previous study because the $\mathrm{Ki}-67$ index has also been used as a proliferation marker to distinguish between the luminal A and luminal B molecular subtypes of ER-positive breast cancer [50]. The feature of homogeneity, which showed the most discriminative power in a similar study [23], also had relatively high prediction performance in either the Ki-67 or tumor grade prediction task in breast cancer. Additionally, Burnside et al. provided effective greatest dimensions and surface areas as important features for prediction [23]. Our findings show that morphological features such as maximum tumor diameter, tumor volume, tumor diameter derived from image slices, mean radius, and diameter from tumor volume have high individual performance (AUC larger than 0.70). Therefore, our results are consistent with previous findings.

The results of this study showed that our multitask learning models improve the prediction of Ki-67 status and tumor grade. This outcome may be explained by the fact that these models

TABLE VI

FEATURE SELECTION FrEQUENCy OF MORE THAN 50\% FOR MUlTitASK LEARNing USing S20 IMAGES

\begin{tabular}{ccc||cc}
\hline \multirow{2}{*}{ Feature } & \multicolumn{2}{c||}{ Ki-67 } & \multicolumn{2}{c}{ Histological grade } \\
\cline { 2 - 5 } & Single task & Multitask & Single task & Multitask \\
\hline Surface area/volume & $144(100 \%)$ & $144(100 \%)$ & $144(100 \%)$ & $144(100 \%)$ \\
Compactness 2 & $144(100 \%)$ & $144(100 \%)$ & $144(100 \%)$ & $132(91.7 \%)$ \\
Information correlation 2 & $144(100 \%)$ & $125(86.8 \%)$ & $115(79.9 \%)$ & $129(89.6 \%)$ \\
Mean radius & $132(91.7 \%)$ & $144(100 \%)$ & $59(41.0 \%)$ & $144(100 \%)$ \\
Information correlation 1 & $78(54.2 \%)$ & $2(1.4 \%)$ & $59(41.0 \%)$ & $0(0 \%)$ \\
Minimum & $2(1.4 \%)$ & $0(0 \%)$ & $144(100 \%)$ & $144(100 \%)$ \\
Difference variance & $0(0 \%)$ & $0(0 \%)$ & $144(100 \%)$ & $144(100 \%)$ \\
Autocorrelation & $0(0 \%)$ & $0(0 \%)$ & $144(100 \%)$ & $119(82.6 \%)$ \\
Energy & $0(0 \%)$ & $107(74.3 \%)$ & $1(0.7 \%)$ & $25(17.4 \%)$ \\
Homogeneity & $0(0 \%)$ & $116(80.1 \%)$ & $0(0 \%)$ & $2(1.4 \%)$ \\
Volume & $0(0 \%)$ & $96(66.7 \%)$ & $0(0 \%)$ & $0(0 \%)$ \\
\hline \hline
\end{tabular}


exploit similar and complementary evaluation of tumor characteristics, hence overcoming the limitations of pathological assessment with noisy data. In both the evaluation tasks we studied, Ki-67 expression and tumor grade, the pathological assessment is important for prognosis and treatment management. Notably, high Ki-67 proliferation status is associated with aggressive growth of breast cancers and hence larger tumor size or higher tumor grade. This measurement is inevitably accompanied by noise; furthermore, tumor proliferation is often spatially heterogeneous [51]. Moreover, in our study, Ki-67 expression status was significantly correlated with tumor grade. These results show that jointly capturing related clinical targets has a crucial impact on the correlated and complementary data. Our experiments show that a multitask prediction approach not only improves the prediction of these indicators but also enhance patient classification for prognostic analysis.

The proposed framework includes combined information from S0, S20, S50 and ADC images, which reflect both morphological and functional tumor biology and could contribute to a net increase in classification performance. Moreover, MRI radiomics analyzed in a multitask way can improve the classifier accuracy for both tasks. The results suggested that the presence of noisy features extracted from heterogeneous tumor images could be partly reduced by the multitask learning method.

Despite some significant findings, the limitations in this study should be addressed. First, the statistical power of this study is limited due to the relatively small number of samples. Further studies with larger sample sizes should be conducted to generalize the findings of this study. Second, we aligned the tumor ROI on the DCE-MRI to that on the ADC images, but the morphological feature values are not identical, hence generating different prediction performances. This limitation is because the ADC map has a relatively low resolution compared with that of DCE-MRI. Third, we collected samples with small tumor sizes (i.e., less than $10 \mathrm{~mm}$ ) for a comprehensive analysis; however, the small size may affect the feature calculation procedure and hence the classification accuracy. Fourth, although we extracted radiomics on subtraction images that may reflect tumor vascularity and angiogenic information, more contrast enhancement features should be included to assess the patterns of kinetic curves, such as the maximum contrast enhancement, time to peak (s) and time at which the maximum enhancement occurs.

\section{CONClusion}

In this paper, we proposed a multitask learning framework for the prediction of breast tumor Ki-67 proliferation status and histological grade. We first analyzed MR series of S0, S20 and S50 and ADC images to evaluate their predictive power. An MTFS and prediction framework was then developed to classify the Ki-67 and tumor grade tasks simultaneously. Multitask learning was conducted using the MR series and DWI along with the proposed multitask framework. The experimental results of breast tumor $\mathrm{Ki}-67$ expression level and grade classification demonstrate the effectiveness of our method. The results are promising for clinical use because patient management decisions are made by simultaneously considering several different biomarkers. Our proposed model could be extended by including additional clinical indicators, such as molecular subtypes, as well as more parametric images, such as T2-weighted imaging, to perform multiple predictions.

\section{REFERENCES}

[1] E. A. Rakha, J. S. Reis-Filho, F. Baehner et al., "Breast cancer prognostic classification in the molecular era: the role of histological grade," Breast Cancer Res, vol. 12, no. 4, pp. 207, 2010.

[2] G. Gasparini, F. Pozza, S. Meli et al., "Breast cancer cell kinetics: immunocytochemical determination of growth fractions by monoclonal antibody Ki-67 and correlation with flow cytometric Sphase and with some features of tumor aggressiveness," Anticancer Res, vol. 11, no. 6, pp. 2015-21, Nov-Dec, 1991.

[3] R. Yerushalmi, R. Woods, P. M. Ravdin et al., "Ki67 in breast cancer: prognostic and predictive potential," Lancet Oncol, vol. 11, no. 2, pp. 174-83, Feb, 2010.

[4] K. I. Kim, K. H. Lee, T. R. Kim et al., "Ki-67 as a predictor of response to neoadjuvant chemotherapy in breast cancer patients," $J$ Breast Cancer, vol. 17, no. 1, pp. 40-6, Mar, 2014.

[5] P. A. Fasching, K. Heusinger, L. Haeberle et al., "Ki67, chemotherapy response, and prognosis in breast cancer patients receiving neoadjuvant treatment," BMC Cancer, vol. 11, pp. 486, Nov 14, 2011

[6] J. R. Brown, M. P. DiGiovanna, B. Killelea et al., "Quantitative assessment Ki-67 score for prediction of response to neoadjuvant chemotherapy in breast cancer," Lab Invest, vol. 94, no. 1, pp. 98106, Jan, 2014.

[7] A. Urruticoechea, I. E. Smith, and M. Dowsett, "Proliferation marker Ki-67 in early breast cancer," J Clin Oncol, vol. 23, no. 28, pp. 7212-20, Oct 01, 2005.

[8] R. Stuart-Harris, C. Caldas, S. E. Pinder et al., "Proliferation markers and survival in early breast cancer: a systematic review and meta-analysis of 85 studies in 32,825 patients," Breast, vol. 17, no. 4, pp. 323-34, Aug, 2008.

[9] G. R. Ricciardi, B. Adamo, A. Ieni et al., "Androgen Receptor (AR), E-Cadherin, and Ki-67 as Emerging Targets and Novel Prognostic Markers in Triple-Negative Breast Cancer (TNBC) Patients," PLoS One, vol. 10, no. 6, pp. e0128368, 2015.

[10] R. M. Warren, L. Pointon, D. Thompson et al., "Reading protocol for dynamic contrast-enhanced MR images of the breast: sensitivity and specificity analysis," Radiology, vol. 236, no. 3, pp. 779-88, Sep, 2005.

[11] L. Zhang, M. Tang, Z. Min et al., "Accuracy of combined dynamic contrast-enhanced magnetic resonance imaging and diffusionweighted imaging for breast cancer detection: a meta-analysis," Acta Radiol, vol. 57, no. 6, pp. 651-60, Jun, 2016.

[12] C. A. Mendez, F. Pizzorni Ferrarese, P. Summers et al., "DCE-MRI and DWI Integration for Breast Lesions Assessment and Heterogeneity Quantification," Int J Biomed Imaging, vol. 2012, pp. 676808, 2012

[13] M. Fan, H. Li, S. Wang et al., "Radiomic analysis reveals DCE-MRI features for prediction of molecular subtypes of breast cancer," PLoS One, vol. 12, no. 2, pp. e0171683, 2017.

[14] H. Li, Y. Zhu, E. S. Burnside et al., "Quantitative MRI radiomics in the prediction of molecular classifications of breast cancer subtypes in the TCGA/TCIA data set," NPJ Breast Cancer, vol. 2, 2016.

[15] E. J. Sutton, B. Z. Dashevsky, J. H. Oh et al., "Breast cancer molecular subtype classifier that incorporates MRI features," $J$ Magn Reson Imaging, vol. 44, no. 1, pp. 122-9, Jan 12, 2016.

[16] M. Fan, T. He, P. Zhang et al., "Diffusion-weighted imaging features of breast tumours and the surrounding stroma reflect intrinsic heterogeneous characteristics of molecular subtypes in breast cancer," NMR Biomed, Dec 15, 2017.

[17] N. M. Braman, M. Etesami, P. Prasanna et al., "Intratumoral and peritumoral radiomics for the pretreatment prediction of pathological complete response to neoadjuvant chemotherapy based on breast DCE-MRI," Breast Cancer Res, vol. 19, no. 1, pp. 57, May 18, 2017.

[18] M. Fan, G. Wu, H. Cheng et al., "Radiomic analysis of DCE-MRI for prediction of response to neoadjuvant chemotherapy in breast cancer patients," Eur J Radiol, vol. 94, pp. 140-147, Sep, 2017. 
[19] S. C. Partridge, Z. Zhang, D. C. Newitt et al., "Diffusion-weighted MRI Findings Predict Pathologic Response in Neoadjuvant Treatment of Breast Cancer: The ACRIN 6698 Multicenter Trial," Radiology, vol. 289, no. 3, pp. 618-627, Dec, 2018.

[20] M. Fan, H. Cheng, P. Zhang et al., "DCE-MRI texture analysis with tumor subregion partitioning for predicting $\mathrm{Ki}-67$ status of estrogen receptor-positive breast cancers," J Magn Reson Imaging, vol. 48, no. 1, pp. 237-247, Dec 8, 2018.

[21] M. Fan, T. He, P. Zhang et al., "Heterogeneity of DiffusionWeighted Imaging in Tumours and the Surrounding Stroma for Prediction of Ki-67 Proliferation Status in Breast Cancer," Sci Rep, vol. 7, no. 1, pp. 2875, Jun 06, 2017.

[22] H. J. Shin, S. H. Kim, H. J. Lee et al., "Tumor apparent diffusion coefficient as an imaging biomarker to predict tumor aggressiveness in patients with estrogen-receptor-positive breast cancer," $N M R$ Biomed, vol. 29, no. 8, pp. 1070-8, Aug, 2016.

[23] E. S. Burnside, K. Drukker, H. Li et al., "Using computer-extracted image phenotypes from tumors on breast magnetic resonance imaging to predict breast cancer pathologic stage," Cancer, vol. 122, no. 5, pp. 748-57, Mar 01, 2016.

[24] L. Martincich, V. Deantoni, I. Bertotto et al., "Correlations between diffusion-weighted imaging and breast cancer biomarkers," Eur Radiol, vol. 22, no. 7, pp. 1519-28, Jul, 2012.

[25] I. Vidic, L. Egnell, N. P. Jerome et al., "Support vector machine for breast cancer classification using diffusion-weighted MRI histogram features: Preliminary study," J Magn Reson Imaging, Oct 16, 2017.

[26] Y. Zhou, J. Xu, Q. Liu et al., "A Radiomics Approach With CNN for Shear-Wave Elastography Breast Tumor Classification," IEEE Trans Biomed Eng, vol. 65, no. 9, pp. 1935-1942, Sep, 2018.

[27] M. Mahrooghy, A. B. Ashraf, D. Daye et al., "Pharmacokinetic Tumor Heterogeneity as a Prognostic Biomarker for Classifying Breast Cancer Recurrence Risk," IEEE Trans Biomed Eng, vol. 62, no. 6, pp. 1585-94, Jun, 2015.

[28] B. Zhang, J. Tian, D. Dong et al., "Radiomics Features of Multiparametric MRI as Novel Prognostic Factors in Advanced Nasopharyngeal Carcinoma," Clin Cancer Res, vol. 23, no. 15, pp. 4259-4269, Aug 1, 2017.

[29] V. S. Parekh, and M. A. Jacobs, "Integrated radiomic framework for breast cancer and tumor biology using advanced machine learning and multiparametric MRI," NPJ Breast Cancer, vol. 3, pp. 43, 2017.

[30] L. Minarikova, W. Bogner, K. Pinker et al., "Investigating the prediction value of multiparametric magnetic resonance imaging at $3 \mathrm{~T}$ in response to neoadjuvant chemotherapy in breast cancer," Eur Radiol, vol. 27, no. 5, pp. 1901-1911, May, 2017.

[31] S. C. Partridge, H. Rahbar, R. Murthy et al., "Improved diagnostic accuracy of breast MRI through combined apparent diffusion coefficients and dynamic contrast-enhanced kinetics," Magn Reson Med, vol. 65, no. 6, pp. 1759-67, Jun, 2011.

[32] J. K. Shin, and J. Y. Kim, "Dynamic contrast-enhanced and diffusion-weighted MRI of estrogen receptor-positive invasive breast cancers: Associations between quantitative MR parameters and Ki-67 proliferation status," J Magn Reson Imaging, vol. 45, no. 1, pp. 94-102, Jan, 2017.

[33] S. S. Chou, E. C. Gombos, S. A. Chikarmane et al., "Computeraided heterogeneity analysis in breast MR imaging assessment of ductal carcinoma in situ: Correlating histologic grade and receptor status," J Magn Reson Imaging, Apr 03, 2017.

[34] T. Wan, B. N. Bloch, D. Plecha et al., "A Radio-genomics Approach for Identifying High Risk Estrogen Receptor-positive Breast Cancers on DCE-MRI: Preliminary Results in Predicting OncotypeDX Risk Scores," Sci Rep, vol. 6, pp. 21394, Feb 18, 2016.

[35] D. Zardavas, A. Irrthum, C. Swanton et al., "Clinical management of breast cancer heterogeneity," Nat Rev Clin Oncol, vol. 12, no. 7, pp. 381-94, Jul, 2015.
[36] A. Argyriou, T. Evgeniou, and M. Pontil, "Convex multi-task feature learning," Machine Learning, vol. 73, no. 3, pp. 243-272, Dec, 2008.

[37] M. Rahim, B. Thirion, D. Bzdok et al., "Joint prediction of multiple scores captures better individual traits from brain images," Neuroimage, vol. 158, pp. 145-154, Sep, 2017.

[38] C. W. Elston, and I. O. Ellis, "Pathological prognostic factors in breast cancer. I. The value of histological grade in breast cancer: experience from a large study with long-term follow-up," Histopathology, vol. 19, no. 5, pp. 403-10, Nov, 1991.

[39] A. Goldhirsch, W. C. Wood, A. S. Coates et al., "Strategies for subtypes--dealing with the diversity of breast cancer: highlights of the St. Gallen International Expert Consensus on the Primary Therapy of Early Breast Cancer 2011," Ann Oncol, vol. 22, no. 8, pp. 1736-47, Aug, 2011.

[40] Q. Yang, L. Li, J. Zhang et al., "Computer-aided diagnosis of breast DCE-MRI images using bilateral asymmetry of contrast enhancement between two breasts," J Digit Imaging, vol. 27, no. 1, pp. 152-60, Feb, 2014.

[41] W. Chen, M. L. Giger, H. Li et al., "Volumetric texture analysis of breast lesions on contrast-enhanced magnetic resonance images," Magn Reson Med, vol. 58, no. 3, pp. 562-71, Sep, 2007.

[42] R. M. Haralick, K. Shanmugam, and I. Dinstein, "Textural Features for Image Classification," IEEE Transactions on Systems, Man, and Cybernetics, vol. SMC-3, no. 6, pp. 610-621, 1973.

[43] Y. Dong, Q. Feng, W. Yang et al., "Preoperative prediction of sentinel lymph node metastasis in breast cancer based on radiomics of T2-weighted fat-suppression and diffusion-weighted MRI," Eur Radiol, vol. 28, no. 2, pp. 582-591, Feb, 2018.

[44] W. Gomez, W. C. Pereira, and A. F. Infantosi, "Analysis of cooccurrence texture statistics as a function of gray-level quantization for classifying breast ultrasound," IEEE Trans Med Imaging, vol. 31, no. 10, pp. 1889-99, Oct, 2012.

[45] P. Brynolfsson, D. Nilsson, T. Torheim et al., "Haralick texture features from apparent diffusion coefficient (ADC) MRI images depend on imaging and pre-processing parameters," Sci Rep, vol. 7, no. 1, pp. 4041, Jun 22, 2017.

[46] V. D. A. Corino, E. Montin, A. Messina et al., "Radiomic analysis of soft tissues sarcomas can distinguish intermediate from highgrade lesions," J Magn Reson Imaging, vol. 47, no. 3, pp. 829-840, Mar, 2018.

[47] J. Zhou, J. Chen, and J. Ye, "MALSAR: Multi-tAsk Learning via StructurAl Regularization," Arizona State University, 2012. http://www.MALSAR.org, 2012.

[48] C. Ciliberto, Y. Mroueh, T. Poggio et al., "Convex learning of multiple tasks and their structure," in Proceedings of the 32nd International Conference on International Conference on Machine Learning - Volume 37, Lille, France, 2015, pp. 1548-1557.

[49] M. Cronin, C. Sangli, M. L. Liu et al., "Analytical validation of the Oncotype DX genomic diagnostic test for recurrence prognosis and therapeutic response prediction in node-negative, estrogen receptorpositive breast cancer," Clin Chem, vol. 53, no. 6, pp. 1084-91, Jun, 2007.

[50] A. Sheri, and M. Dowsett, "Developments in Ki67 and other biomarkers for treatment decision making in breast cancer," Ann Oncol, vol. 23 Suppl 10, pp. x219-27, Sep, 2012.

[51] A. S. Coates, E. P. Winer, A. Goldhirsch et al., "Tailoring therapies-improving the management of early breast cancer: St Gallen International Expert Consensus on the Primary Therapy of Early Breast Cancer 2015," Ann Oncol, vol. 26, no. 8, pp. 1533-46, Aug, 2015. 